\title{
Questes
}

\section{Amour et pèlerinage dans quelques romans d'aventure}

Aurélie Houdebert

\section{(2) OpenEdition}

Journals

\section{Édition électronique}

URL : http://journals.openedition.org/questes/1410

DOI : 10.4000/questes. 1410

ISSN : 2109-9472

Éditeur

Les Amis de Questes

\section{Édition imprimée}

Date de publication : 15 novembre 2011

Pagination : 35-49

ISSN : 2102-7188

\section{Référence électronique}

Aurélie Houdebert, « Amour et pèlerinage dans quelques romans d'aventure », Questes [En ligne], 22 | 2011, mis en ligne le 01 janvier 2014, consulté le 22 avril 2019. URL : http:// journals.openedition.org/questes/1410; DOI : 10.4000/questes.1410 


\title{
Amour et pèlerinage dans quelques romans d'aventure
}

\author{
Aurélie HOUDEBERT
}

Le pèlerinage, souvent associé à la croisade dans la geste de Charlemagne où les chevaliers chrétiens empruntent la route de Compostelle pour communier et conquérir dans un même mouvement, connaît une certaine fortune littéraire en tant que motif narratif dans les romans des $\mathrm{XII}^{\mathrm{e}}$ et $\mathrm{XIII}^{\mathrm{e}}$ siècles. Topiques et néanmoins malléables, les épisodes de pèlerinage s'offrent en effet comme des ressorts narratifs précieux dans des textes mettant en scène des héros qui progressent et s'accomplissent à travers quêtes et épreuves variées.

Écartant les romans arthuriens qui préfèrent souvent, dans la formation de leur héros, la rencontre avec un ermite à la prise de bourdon, je voudrais confronter ici la manière dont quelques romans d'aventure des $\mathrm{XII}^{\mathrm{e}}$ et $\mathrm{XIII}^{\mathrm{e}}$ siècles exploitent le motif du pèlerinage. Floire et Blanchefleur, Amadas et Ydoine, L'Escoufle de Jean Renart, sont trois représentants du «nouveau roman » médiéval $^{1}$, ce genre romanesque qui échappe à la classification en trois «matières", - France, Rome, Bretagne - définie par Jean Bodel ${ }^{2}$. Peu portés sur la féerie, ces récits ont ceci en commun qu'ils mettent sur les routes des personnages mus par l'amour : leurs héros sont des amants qui, se trouvant séparés et éloignés par le destin, entreprennent des quêtes parallèles sur les routes d'Occident ou d'Orient pour se rejoindre. Les romans s'achèvent lorsque les couples

\footnotetext{
1 Nous reprenons ici l'expression proposée par Jean Dufournet. Cf. JEAN RENART, Guillaume de Dole ou le Roman de la Rose, Jean Dufournet (trad.), Paris, Champion, «Traductions des Classiques français du Moyen Âge », 27, 1988, p. 136-139.

2 Les premiers vers de la Chanson des Saisnes distinguent en effet la geste de Charlemagne (matière de France), les romans antiques (matière de Rome) et les romans arthuriens (matière de Bretagne). Cf. JEHAN BoDEL, La Chanson des Saisnes, Annette BRASSEUR (éd.), Genève, Droz, «Textes Littéraires Français », 369, 1989, v. 6-11.
} 
sont reformés et qu'ils obtiennent la reconnaissance des sociétés qui les avaient initialement disjoints ${ }^{3}$.

Dans ces récits, le motif du pèlerinage subit certaines distorsions par rapport à son modèle épique. Ces trois romans remotivent le topos en l'associant à la thématique amoureuse, lui ôtant du même coup, mais à des degrés divers, sa dimension spirituelle. Les pèlerinages deviennent surtout des ressorts romanesques de choix, dans des récits entièrement construits sur les notions de mouvement, de cheminement et de quête. Dans ces trois romans, enfin, le pèlerinage apparaît dans sa dimension concrète : il est l'occasion pour les auteurs d'insister sur la matérialité du parcours, sur les dangers des routes, sur les conditions de subsistance et d'hébergement du pèlerin. Car ce qu'éprouvent les amants à travers ces pèlerinages, c'est d'abord la force de leur amour face aux aléas d'un monde qui leur est hostile.

\section{Routes et détours de l'amour et de la foi (Floire et Blanchefleur)}

Dans Floire et Blanchefleur ${ }^{4}$, l'épisode du pèlerinage est tout à fait secondaire ; il est relégué aux marges du récit principal car il appartient à

\footnotetext{
${ }^{3}$ Floire et Blanchefleur et Amadas et Ydoine présentent une structure narrative et des thèmes qui correspondent assez précisément au type de romans défini par Myrrha Lot-Borodine et, plus récemment, par Jean-Jacques Vincensini : les romans idylliques. L'Escoufle de Jean Renart s'y rattache également par certains aspects, comme l'a démontré Marion Vuagnoux-Uhlig, quoique l'appellation la plus fréquente pour ce roman reste celle de « roman réaliste ». Christine Ferlampin-Acher propose pour sa part l'appellation de « roman du troisième type » pour ces récits inclassables, par opposition aux romans antiques et arthuriens. Pour toutes ces définitions, voir Myrrha Lot-Borodine, Le Roman idyllique au Moyen Âge, Paris, Auguste Picard, 1913 ; Le Récit idyllique. Aux sources du roman moderne, Jean-Jacques VINCENSINI et Claudio GALDERISI (dir.), Paris, Classiques Garnier, «Recherches Littéraires Médiévales », 2, 2009 ; Marion VuagnouX-Uhlig, Le Couple en herbe. Galeran de Bretagne et L'Escoufle à la lumière du roman idyllique médiéval, Genève, Droz, «Publications Romanes et Françaises », 245, 2009 ; Christine FERLAMPIN-ACHER, « Féerie et idylles : des amours contrariées », in Michelle SzKILNIK (dir.), Idylle et récits idylliques à la fin du Moyen Âge, Cahiers de Recherches Médiévales et Humanistes, 20 (2010).

${ }^{4}$ Le Conte de Floire et Blanchefleur, Jean-Luc LECLANCHE (éd. et trad.), Paris, Champion, «Classiques français du Moyen Âge », 2, 2003.
} 
ce que l'on pourrait appeler les «enfances » des héros, c'est-à-dire à l'histoire de leurs parents. La mère de Blanchefleur, enceinte, apparaît pour la première fois dans le récit comme une pèlerine en route pour Saint-Jacques. Accompagnée de son père, dont on nous dit simplement qu'il est un chevalier français, elle s'est vouée au saint après la mort de son $\mathrm{ami}^{5}$. Privée d'amour, la dame s'est tournée vers le saint apôtre, et le pèlerinage correspond donc aussi pour elle à un renoncement au monde. Nous rencontrons la jeune veuve alors qu'elle atteint l'ultime étape de son voyage, en Galice, entourée d'un groupe de pèlerins ${ }^{6}$. Ainsi, au seuil même du roman, l'amour met en mouvement des personnages, les propulse sur les routes, et le pèlerinage apparaît comme l'une des modalités de ce mouvement amené à se réaliser sous d'autres formes dans la suite du récit ${ }^{7}$.

Mais ce qui surprend dans le pèlerinage de Floire et Blanchefleur, et qui donne le ton de ce roman d'aventures, c'est qu'il tourne court. Les pèlerins chrétiens sont victimes d'un raid sarrasin qui met brutalement fin à leur marche. Pris dans une embuscade, les pèlerins se défont de leurs biens sans résistance; seul le chevalier français s'oppose aux assaillants pour défendre sa fille, objet de la convoitise des Sarrasins. En trois vers d'une efficacité redoutable, le poète relate le meurtre du père et le rapt de la fille ${ }^{8}$. Le long chemin entrepris par la mère de l'héroïne est donc interrompu et le vœu prononcé ne sera pas réalisé. Ni la Galice ni la France ne seront plus

\footnotetext{
5 «En la compaigne ot un François, / Chevalier et preu et courtois, / Qui au baron saint Jake aloit ; / Une soie fille i menoit / Qui a l'apostle s'ert vouee / Ains qu'ele issist de sa contree / Por son ami qui mors estoit, / De cui remese ençainte estoit. » (Le Conte de Floire et Blanchefleur, éd. cit., v. 93-100).

${ }^{6}$ Notons que cet épisode du pèlerinage, absent du conte oriental dont le roman français s'inspire, est présent dans tous les manuscrits, ce qui souligne son importance pour les remanieurs occidentaux malgré l'espace réduit qu'il occupe dans le texte.

${ }^{7} \mathrm{Au}$ cheminement de la mère de Blanchefleur répondront les errances de Floire : tous deux cherchent sur les routes l'annulation de l'insupportable souffrance que représente la perte, effective ou fantasmée, de l'être aimé.

${ }^{8}$ «De lui ne caut a aus vif prendre / Ains l'ocïent, sel laissent mort / Et sa fille mainent au port. » (Le Conte de Floire et Blanchefleur, éd. cit., v. 102-104).
} 
évoquées dans le roman, qui quitte les voies de la Chrétienté pour s'engager sur celles des royaumes musulmans d'Espagne, avant de nous entraîner en Égypte.

La grande habileté du conteur est d'utiliser le pèlerinage à la fois pour lancer la dynamique narrative et pour ancrer son récit dans une certaine réalité. La rencontre entre la mère de l'héroïne - la pèlerine chrétienne - et le père du héros - le roi sarrasin qui a commandité l'enlèvement - se fait sur un mode particulièrement violent, mais elle semble relever d'une conjonction d'événements qui n'a rien d'exceptionnel dans ce contexte des routes de pèlerinages. Les opérations de pillage sont présentées comme un moyen tout à fait naturel pour les souverains maures d'enrichir leur royaume et les pèlerins sont la cible idéale, banale, de ce genre d'expédition. Le roi Félis désigne la route de Compostelle par l'expression «ces chemins », ce qui renvoie à un référent suffisamment connu pour ne pas nécessiter d'explication ${ }^{9}$. L'attaque de pèlerins semble bien être une réalité quotidienne, et les voyageurs y sont préparés : ils tendent leurs bourses et échappent ainsi à la mort ${ }^{10}$. La pratique de l'enlèvement de femmes est là encore présentée comme fréquente, et le tort du chevalier assassiné est de ne pas avoir accepté de considérer sa fille comme un bien monnayable. Elle sera pourtant accueillie comme un joyau de prix par le roi Félis à qui elle est menée ${ }^{11}$.

Nous touchons ici au trait le plus original dans le traitement du motif du pèlerinage : le choix de la focalisation opéré par le narrateur. Toute la scène est narrée du point de vue du roi païen et de ses chevaliers. Le lecteur

\footnotetext{
9 «Alés lassus en ces chemins / Gaitier por reuber pelerins !» (ibid., v. 82-83). Félis se trouvant sur son navire, au large de la Galice, au moment où il envoie ses mercenaires attaquer des pèlerins, la valeur du démonstratif n'est certainement pas déictique.

${ }^{10}$ «Et li pelerin se defalent / De combatre tot li plusor, / Lor avoir tendent por paour. » (ibid., v. 89-91).

${ }^{11}$ Devant la grande beauté de la captive chrétienne, le roi sarrasin décide d'en faire présent à sa femme et la ramène donc comme un trésor de guerre, ou plutôt comme un souvenir de voyage (ibid., v. 105-112).
} 
suit en effet les pas de Félis et de ses hommes et la route du pèlerinage n'est que furtivement aperçue, du haut de la montagne où les Sarrasins tendent leur embuscade. Le cœur de l'intrigue se déroulera en Espagne, d'où vient le roi et où il retourne avec sa captive, où naîtront et règneront les héros du roman. Le point de vue de la mère de Blanchefleur n'est jamais pris en compte dans ces premiers vers du roman; aucune plainte n'émane d'elle et elle se contente d'être menée en Espagne, c'est-à-dire de dévier malgré elle et pour toujours de la route qu'elle s'était tracée. En somme, il s'agit ici d'un détournement de pèlerinage, qui ne semble émouvoir ni le narrateur, ni la pèlerine elle-même.

On pourrait être tenté de voir dans cet épisode un procédé narratif habile et purement technique pour amener le lecteur occidental d'un univers familier, celui des routes de Saint-Jacques partant de France, à un monde plus exotique, celui des royaumes musulmans d'Espagne. Mais, à l'échelle de l'ensemble du roman, ce pèlerinage avorté prend un sens plus symbolique. Le roman s'ouvre en effet sur une double rupture : la mort du père de Blanchefleur et l'inachèvement du pèlerinage de sa mère sont deux violences faites à l'amour et à la foi. Privée d'amour et détournée de la route sainte, la jeune captive se résignera à son sort, élevant sa fille dans les préceptes chrétiens en terre sarrasine. Or, ces deux privations initiales de la mère seront réparées à la fin du roman par le bonheur conjugal de la fille et par l'avènement du christianisme en terre païenne. Floire et Blanchefleur sont en effet réunis après de nombreuses errances, et tout le royaume se convertit au christianisme en même temps qu'il célèbre l'amour ${ }^{12}$. L'amour brisé et le pèlerinage interrompu trouvent ainsi conjointement leur achèvement dans le mariage et le baptême, une génération plus tard.

${ }^{12}$ Ibid., v. 3307-3318. 


\section{Amant parfait, parfait pèlerin (L'Escoufle)}

Le pèlerinage occupe un espace narratif plus important dans $L^{\prime}$ Escoufle $^{13}$, où il intervient au cœur même de l'intrigue amoureuse. Le dernier tiers du roman, consacré aux errances du héros en quête de son amie, comporte en effet plusieurs péripéties liées au pèlerinage. Aélis et Guillaume, promis l'un à l'autre dès leur plus jeune âge, sont séparés par l'empereur de Rome, père de la jeune fille ; ils décident de prendre la fuite ensemble pour rejoindre la Normandie où Guillaume entend reprendre possession de ses terres. Près de Toul, en Lorraine, ils sont de nouveau séparés par un malheureux hasard : un escoufle, un milan, dérobe l'anneau d'Aélis et Guillaume, furieux, poursuit l'oiseau, laissant dans la clairière son amie endormie. À son retour, Aélis a disparu. Les deux amants, interprétant chacun de façon erronée la disparition de l'autre, vont emprunter des chemins opposés et il leur faudra sept années pour se retrouver.

Aélis emprunte des chemins profanes, selon une logique très pragmatique : elle se rend d'abord dans la ville la plus proche, Toul, pour y reprendre des forces avant de gagner la Normandie, où elle espère retrouver Guillaume. Déçue et démunie après ce voyage, elle décide de s'établir quelque part pour gagner sa vie et attendre un sort meilleur. Elle se rend donc à Montpellier où elle fonde un atelier de broderie avec sa compagne de fortune, Isabelle. C'est une intrigue amoureuse parfaitement courtoise entre deux personnages secondaires qui la conduira finalement à Saint-Gilles, dans le Gard $^{14}$. Rien de spirituel donc dans ce trajet vers

\footnotetext{
${ }^{13}$ JEAn RENART, L'Escoufle, roman d'a venture, Franklin SwEETSER (éd.), Genève, Droz, «Textes Littéraires Français », 211, 1974. L'éditeur se base sur le manuscrit Paris, BnF, Arsenal 6565.

${ }^{14}$ Une ceinture brodée par Aélis pour la dame de Montpellier et donnée à l'amant de celle-ci, le comte de Saint-Gilles, éveille la jalousie de l'épouse de ce dernier ; le comte
} 
Saint-Gilles, pourtant connu comme un haut lieu de pèlerinage au $\mathrm{XIII}^{\mathrm{e}}$ siècle ${ }^{15}$.

Guillaume est lui aussi conduit dans sa quête à la fois par son raisonnement logique et par le hasard. Il rebrousse chemin vers Rome car il pense qu'Aélis a été enlevée par son père et qu'elle est détenue dans sa ville natale. Puis, sans que l'on sache exactement dans quelles circonstances ni à quel moment précis de son errance, il se rend à SaintJacques-de-Compostelle, où nous le retrouvons déjà établi depuis quelques temps auprès d'un bourgeois. Un témoin lui indique que son amie a été vue à Toul, où il se rend alors, mais dont il repart aussitôt, déçu et désorienté. Empruntant le «grand chemin de France » en désespoir de cause ${ }^{16}$, il finit par envisager de se rendre à Saint-Gilles pour prier le saint ${ }^{17}$. C'est là, après plusieurs péripéties, que les deux amants se retrouveront.

Il apparaît de manière troublante que le chemin emprunté par Guillaume recoupe de grandes routes de pèlerinage (Rome, Saint-Jacques, Saint-Gilles). Mais la coïncidence entre les routes de la quête et les routes de pèlerinage semble fortuite, du moins pour les deux premiers trajets ${ }^{18}$. Si le héros se rend à Rome, c'est parce qu'il pense qu'Aélis, romaine, y a été ramenée de force. Aucune mention de visite aux sanctuaires romains, ni de dévotions rendues à saint Pierre. Tout au plus Guillaume a-t-il

fait venir les brodeuses chez lui afin d'apaiser la colère de sa femme (Ibid., v. 5900-6144).

15 Voir par exemple Marcel et Pierre-Gilles GIRAULT, Visages de pèlerins au Moyen Âge: les pèlerinages européens dans l'art et l'épopée, Saint-Léger-Vauban, Zodiaque, «Visages du Moyen Âge », 3, 2001.

16 «[... A quel main prendrai gié / Le grant cemin qui vait en France?» (JEAN RENART, L'Escoufle, éd. cit., v. 6456-6457).

${ }^{17}$ « [...] Ja l'ai jou quisse ja / Dusqu'a S. Jake et dusqu'a Rome ; / Mais al saint qui ne faut nul home / Ki de cuer li prit k'il ne l'oie, / A celui promet jou la voie / Que jou requerrai a sa vile. » (ibid., v. 6478-6483). Guillaume évoque dans ces quelques vers les trois grandes étapes de son errance, qui correspondent à trois des grands lieux de pèlerinage occidentaux.

18 En effet, la motivation de Guillaume n'appartient pas aux grandes catégories dégagées dans l'introduction au thème du bulletin. Voir dans ce bulletin l'introduction de Magali CHEYNET, p. 9-23. 
probablement séjourné dans un hôpital, mais en qualité de malade, pas en tant que pèlerin ${ }^{19}$. De même, le voyage et le séjour à Compostelle semblent dictés par le désir de retrouver la femme aimée et par les contingences matérielles. Saint-Jacques étant un lieu de passage privilégié, Guillaume espère probablement obtenir des nouvelles d'Aélis, ce qui se produit effectivement. Le séjour est aussi l'occasion pour le jeune homme d'amasser des gains afin de poursuivre sa quête, et on peut mettre en parallèle l'emploi du héros dans une auberge pour pèlerins avec l'activité de broderie exercée par Aélis : il faut se maintenir en vie et trouver les moyens matériels de chercher l'autre.

C'est cette dimension concrète, cette attention au travail et à l'activité humaine dans des espaces quotidiens qui a retenu l'attention de la plupart des critiques, et qui a valu au roman la qualification de «réaliste ${ }^{20}$. L'historien appréciera en effet la foule de détails sur les difficultés rencontrées sur les routes de pèlerinage et sur la vie de ces villes entièrement organisées autour des sanctuaires. À SaintJacques-de-Compostelle comme à Saint-Gilles, Guillaume se fait employer dans des auberges qui accueillent les pèlerins. Les mentions de l'activité fébrile de ces maisons et de l'argent qui y est drainé abondent. Plusieurs vers sont ainsi consacrés aux gains que le héros amasse grâce au cumul de son salaire et des pourboires versés par les bourgeois de passage ${ }^{21}$. Par ailleurs, les scènes de rue et d'auberge dans ces villes sont très vivantes et

19 «Puis li prist .i. [si] grans malages / Dont il gut près d'un an a Rome. » (JEAN RENART, L'Escoufle, éd. cit., v. 6178-6179).

${ }^{20}$ L'article fondateur sur ce sujet est celui de Rita LEJEUNE, «Jean Renart et le roman réaliste au XIII ${ }^{\mathrm{e}}$ siècle », Gründriss der romanischen Literaturen des Mittelalters, IV/1, Heidelberg, C. Winter, 1978, p. 400-453.

${ }^{21}$ Une longue scène relate l'établissement du contrat d'embauche de Guillaume chez le bourgeois de Saint-Gilles, puis les conseils d'un autre bourgeois l'incitant à demander un salaire plus élevé en raison de la manne financière que représentent les pèlerins, et enfin l'accumulation des pourboires reçus et épargnés par Guillaume après quelques semaines de travail seulement (JEAN RENART, L'Escoufle, éd. cit., v. 6572-6611). 
mêlent des registres variés : ainsi le conflit qui oppose Guillaume et un pèlerin lorrain au sujet d'une mule est-il particulièrement cocasse ${ }^{22}$.

Les pèlerinages sont donc l'occasion pour le romancier de multiplier les notations pittoresques, mais ils constituent surtout de formidables ressorts romanesques. Le pèlerinage permet en effet de faire alterner séquences de marche et longues étapes, mimant ainsi le mouvement de la quête amoureuse, faite d'élan et d'attente, d'espérance et de doute. Les notations temporelles sont nombreuses dans ces épisodes, qui rendent compte de l'épaisseur du temps qui passe, temps de souffrance pour les amants séparés : le temps du voyage, le temps du séjour sont véritablement éprouvés par Guillaume ${ }^{23}$.

C'est enfin cette dimension d'épreuve qui place le pèlerinage au cœur du dispositif romanesque. Car la quête amoureuse utilise le langage de la quête spirituelle particulière que constitue le pèlerinage. En effet, si l'on dépasse la logique du hasard et celle de la nécessité matérielle, qui semblent suffire à justifier les déplacements des héros, on constate que leur progression l'un vers l'autre est également le fruit d'un plan bien défini, dont l'artisan est tantôt le dieu païen de l'Amour, tantôt le Dieu chrétien. Comme dans le pèlerinage, la pérégrination des amants est une mise à l'épreuve de la force de leur engagement, et l'idéal amoureux qui se dégage de cette expérience douloureuse se construit bien plus sur le modèle de la foi chrétienne que sur celui de l'amour courtois. L'expérience concrète de la souffrance physique liée à la marche met le pèlerin dans les pas du Christ

\footnotetext{
${ }^{22}$ Le pèlerin se fait en effet agresser par Guillaume, qui a reconnu dans la monture du bon bourgeois la mule de son amie; le voyageur se plaint au prêtre du sanctuaire, qui abandonne la garde du trésor et sort de l'église pour venir calmer la querelle. Une foule de badauds s'est assemblée sur la place et assiste aux lamentations émues de Guillaume sur sa mule avant la réconciliation des deux parties en conflit (ibid., v. 6206-6305).

${ }^{23}$ Lorsque nous retrouvons Guillaume à Compostelle, il nous est présenté comme un héros usé par la quête et par l'attente. Les indications de durée participent pleinement au pathétique du récit: Guillaume a cherché Aélis «.vii. ans tous plains », une maladie l'a retenu à Rome «pres d'un an », la nécessité l'a contraint à rester «toute une saison » chez le bourgeois de Compostelle, soit « demi an et .iii. mois » (ibid., v. 6184-6205).
} 
et l'amant sur la voie du véritable amour. C'est pourquoi Jean Renart place Guillaume et Aélis au-dessus de ces amants mythiques que sont Tristan et Yseut ou Pyrame et Thisbé, aveuglés par leur désir et prompts au désespoir lorsqu'ils sont séparés par le destin ${ }^{24}$. Dans un curieux syncrétisme, le romancier mêle Fortune, Amour et Dieu dans une même intention, entièrement tournée vers l'accomplissement d'un bonheur terrestre.

Ainsi se comprend mieux le parcours de Guillaume d'un lieu de pèlerinage à l'autre : à Rome et à Saint-Jacques, sa foi n'intervient pas car il emprunte les routes de pèlerinage malgré lui. Mais lorsqu'il perd confiance en ses propres ressources, et qu'il abandonne par là même tout orgueil, le héros se tourne explicitement vers saint Gilles dans une attitude de requête propre au pèlerin. C'est avec sincérité et ardeur qu'il rend ses dévotions au saint dans la cathédrale où, pour la première fois, nous pénétrons avec lui ${ }^{25}$. Alors seulement l'ensemble de son parcours prend-il sens et les coïncidences qui le mènent progressivement vers son amie peuvent-elles s'interpréter comme des signes de Dieu : ainsi Guillaume, enfin ouvert à la Providence, relit-il a posteriori l'épisode de la mule retrouvée à Compostelle comme une intervention de l'apôtre Jacques ${ }^{26}$. Si les amants sont finalement guidés l'un vers l'autre, c'est grâce à l'intervention conjointe des saints et de Dieu lui-même, comme le comprend Guillaume et comme l'affirme de plus en plus clairement le

${ }^{24}$ L'auteur se livre à une analyse comparée du destin de trois amants exemplaires : Tristan agit de façon inconsidérée car sa volonté est anéantie par la «force»du breuvage magique ( «Dont fu ce force : force voire! / K'il n'i ot onques point de grace », ibid., v. 6358-6359); Pyrame est un modèle d'amant parfait, mais Jean Renart ne sait se prononcer sur sa conduite : est-ce vraiment l'amour, ou bien la folie qui le pousse à se donner la mort («Por ce ne sai jou que j'en die, / Se ce fu folie u amors. », ibid., v. 6364-6365) ? Guillaume, lui, résiste à la tentation du suicide et fait preuve de plus de constance que ses ancêtres littéraires : il ne désespère jamais et conserve sa foi intacte, même dans l'épreuve («Mais sospechons et desconfors / Ne la grant paine qu'il en a / Ne le pot onques dusque la / Mener qu'il se vausist ocire », ibid., v. 6384-6387).

25 «Il va son veu et sa promesse / Rendre au saint et s'offrande faire, / Et si le requiert de l'afaire / Dont il a tant mal et anui. » (ibid., v. 6494-6497).

${ }^{26}$ «Mesires sains Jakes m'en ra / Mostré son mur, soie merci. » (ibid., v. 6424-6425). 
narrateur à mesure que le récit progresse ${ }^{27}$. La loi d'amour et les chemins du hasard rencontrent ainsi, sans aucun didactisme, la morale chrétienne, grâce au thème central du pèlerinage.

\section{Faux pèlerinage et vraie amors ${ }^{28}$ (Amadas et Ydoine)}

Dans Amadas et Ydoine, c'est aussi un amour pur, éprouvé par la quête et consacré par le mariage, qui est glorifié. Mais la morale sociale et religieuse y est préservée au prix de bien des compromis. En effet, les deux amants éponymes sont séparés par le père de la jeune fille, qui donne à celle-ci un époux digne de son rang, le comte de Nevers. L'obstacle majeur pour les amants est donc un mari, que seule la ruse d'Ydoine permettra de contourner sans atteinte à la morale.

Le pèlerinage joue un rôle important dans la délicate entreprise de justification des manœuvres amoureuses des personnages. Ydoine, apprenant qu'Amadas se trouve à Lucques, où il se comporte comme un misérable fou depuis qu'il a perdu son amie, décide d'entreprendre un pèlerinage à Rome afin de pouvoir rejoindre son amant sur le trajet. Rome est effectivement une destination rêvée pour prétexter une étape à Lucques, en Toscane. La comtesse doit donc obtenir l'accord de son époux et se livre à un savant discours de manipulation afin de justifier ce départ ${ }^{29}$. La rhétorique religieuse domine: la comtesse se réclame constamment de Dieu. Elle prétend qu'un intermédiaire divin lui est apparu en songe pour

\footnotetext{
${ }^{27}$ Ce sont d'ailleurs les larmes de compassion versées par Aélis au récit des infortunes de Guillaume qui permettent la reconnaissance. Le parcours des deux amants, comme celui des pèlerins, est donc bien celui d'une purification qui passe par un abandon de soi entre les mains de Dieu, condition nécessaire à la pleine réalisation d'un amor évoluant en caritas.

28 «Mult par ot en li vraie amie / Li quens et ele vrai ami / En lui tous jors, tant com vesqui. » (Amadas et Ydoine, John R. REINHARD (éd.), Paris, Champion, «Classiques français du Moyen Âge », 51, 1926, v. 7882-7884).

${ }^{29}$ «Piteusement pleure et o lermes, / Et dist : [...]» (ibid., v. 2931-2966).
} 
l'exhorter à prendre le bourdon ${ }^{30}$, topos que l'on connaît bien depuis la Chronique du Pseudo-Turpin ${ }^{31}$. Elle avance également une motivation tout à fait crédible pour un pèlerinage, la guérison d'une maladie par les dévotions rendues à un saint ${ }^{32}$. Convaincu, le bon mari organise aussitôt l'expédition de sa femme.

On frôle donc le fabliau dans cet épisode de faux pèlerinage ${ }^{33}$, et pourtant jamais le poète ne condamne explicitement son héroïne sans tenter en même temps de justifier ses agissements. Ydoine n'est pas une bourgeoise en mal de plaisir ; elle est certes une mal mariée mais, ce qui la pousse à la ruse, c'est la pureté de son engagement amoureux envers Amadas. L'union présentée comme naturelle et juste entre ces deux amants admet le recours au mensonge, parfaitement naturel aussi chez une fille d'Ève. Les paroles à double entente autour des notions de maladie et de guérison, qui accompagnent le motif du pèlerinage, rapprochent Ydoine $\mathrm{d}^{\prime}$ Yseut $^{34}$, cette folle amante condamnée par Jean Renart mais pardonnée

${ }^{30}$ Ydoine n'en est d'ailleurs pas à sa première mystification en matière de songe prémonitoire : avec l'aide de trois sorcières, elle a précédemment suggéré à son époux un songe pour le mettre en garde contre les conséquences mortelles d'une consommation du mariage. La comtesse a ainsi réussi à préserver sa virginité.

${ }^{31}$ Le songe de Charlemagne mettant en scène saint Jacques ordonnant à l'empereur de fonder le pèlerinage de Compostelle est en effet abondamment repris par la chanson de geste et l'iconographie à la suite du Pseudo-Turpin. Ydoine en fait un argument décisif : «[...] en avision / M'est par trois termes aparu / Uns biaus hom flouri et canu [...]» (ibid., v. 2950-2952).

${ }^{32}$ C'est en effet l'une des motivations les plus fréquentes du pèlerinage : « [...] g’irai / Mult tost a Diu ou je garrai [...] » (ibid., v. 2957-2958).

${ }^{33}$ Plusieurs fabliaux grivois fondent en effet leur comique sur la polysémie des mots « maladie », « guérison », « soin », comme La Saineresse, in Recueil général et complet des fabliaux des XIII ${ }^{\mathrm{e}}$ et XIV $\mathrm{X}^{\mathrm{e}}$ siècles imprimés ou inédits, Anatole de MONTAIGLON et Gaston RAYNAUD (éd.), Paris, Librairie des bibliophiles, 1872-1890, t. I, p. 289-293. Le motif du songe suggéré par une femme à son mari pour le tromper en toute quiétude est présent dans le fabliau Des tresces, in Recueil général et complet des fabliaux des XIII et $\mathrm{XIV}^{\mathrm{e}}$ siècles..., éd. cit., t. IV, p. 67-81 et, de façon plus développée encore, dans sa variante De la dame qui fist entendant son mari qu'il sonjoit, in Recueil général et complet des fabliaux des $\mathrm{XIII}^{\mathrm{e}}$ et XIV $\mathrm{X}^{\mathrm{e}}$ siècles..., éd. cit., t. V, p. 132-142.

${ }^{34}$ On songe en effet aux subtilités de langage d'Yseut, jurant par exemple n'avoir connu d'autre homme entre ses cuisses que le mendiant qui l'a portée pour traverser le gué. 
en tant que femme par l'auteur du Roman d'Amadas: Ydoine se prétend malade pour dissimuler son mal d'amour, et elle espère obtenir à Rome une guérison qui se produira en réalité à Lucques dans les bras de son amant. Le terme de «guérison » apparaît chargé de cette ambiguïté plaisante dans la plupart de ses occurrences.

Instrument de l'amour profane, le pèlerinage ne facilite pas pour autant d'emblée la formation du couple. Au contraire, il apparaît plutôt comme le cadre d'une série d'épreuves qui ne cessent de repousser le moment de l'union des corps et des âmes. Réactivant les éléments traditionnellement associés au motif du pèlerinage, l'auteur fait longuement souffrir ses pèlerins, fussent-ils de comédie. La comtesse de Nevers découvre les dangers de la route, malgré l'escorte nombreuse que lui octroie son époux. Cinq chevaliers l'encadrent, dont un vieillard qui tient les rênes de son palefroi ${ }^{35}$. Écuyers et serviteurs divers assurent la sécurité et le confort de la comtesse tout en veillant sur le somptueux trésor transporté dans des coffres afin de couvrir les frais du voyage. Ces précautions n'empêchent pas Ydoine de subir un enlèvement, comme la mère de Blanchefleur. Un mystérieux chevalier surgit d'un bois, neutralise facilement la garde rapprochée de la comtesse et prend cette dernière en croupe avant de partir au galop sous le regard impuissant du reste de l'escorte. Il faudra l'intervention heureuse d'Amadas faisant route en sens inverse à la rencontre de la pèlerine pour que l'héroïne soit sauvée, pour cette fois du moins ${ }^{36}$.

Cf. Beroul, Le Roman de Tristan, Ernest Muret (éd.), Paris, Champion, « Classiques français du Moyen Âge », 12, 2000 (1 ${ }^{\text {ère }}$ éd. 1957), v. 4197-4216.

35 «Par la rice resne la tient / Uns vius chevaliers qui la guie, / Car mult souvent ont en baillie / Teus gens les dames a guier / Et a conduire et a mener, / Que mains est doutés hom de jors / Que li jovenes en toutes cors.» (Amadas et Ydoine, éd. cit., v. 4607-4624). On retrouve ici le soupçon d'immoralité qui pèse sur les pèlerines au Moyen Âge.

${ }^{36}$ Ibid., v. 4614-4675. 
Fertile en péripéties, le long épisode du faux pèlerinage est donc l'occasion pour le héros d'éprouver son amour mais aussi, et contre toute attente, de purifier cet amour sous le regard de Dieu. La dernière péripétie liée au pèlerinage prend en effet une tournure sacrificielle : à peine revenue de Rome, fraîchement rescapée d'un rapt, Ydoine croit enfin pouvoir jouir de son amant dans l'auberge de Lucques où elle fait de nouveau étape. Mais lors du repas, elle est prise d'un mystérieux mal qui l'emporte en quelques heures. Il faudra à l'amant fidèle affronter la mort, protéger le corps de sa bien-aimée réclamé par un rival de l'Autre Monde et obtenir, par un combat nocturne avec un démon, son retour à la vie ${ }^{37}$. Par ses exploits contre les ennemis de Dieu, le héros rachète les mensonges d'Ydoine et mérite en même temps son amour ${ }^{38}$. Forts de cet amour ainsi reconnu par le Ciel, et au prix encore de quelques manipulations oratoires d'Ydoine auprès de son époux, les amants obtiendront légitimement le mariage.

On voit donc à travers ces exemples que le roman d'aventure exploite toutes les ressources du motif du pèlerinage, en renouvelant sensiblement sa signification et son écriture. S'intégrant parfaitement à la structure de ces romans de la quête et de l'errance, le pèlerinage devient une épreuve sur le chemin d'amour, ouvrant la voie à une symbolique qui met en parallèle cultes chrétiens et dévotions amoureuses. Mais la dimension spirituelle n'est finalement pas écartée de ces épisodes dans ces trois romans d'amour. Elle est moins lisible et apparaît parfois comme une simple métaphore des épreuves par lesquelles l'engagement amoureux se mesure, mais elle participe aussi en profondeur au projet idéologique

\footnotetext{
${ }^{37}$ Ibid., v. 5650-6461.

${ }^{38}$ Ydoine se révèle en effet une amante bien plus entreprenante que son compagnon. Par amour pour Amadas, elle met en péril le salut de son âme par des discours et des pratiques proches du blasphème et de l'hérésie. Les agissements de la jeune femme traduisent la force de son amour, mais témoignent aussi d'une forme de conscience supérieure de la miséricorde divine.
} 
commun à tous ces textes : il s'agit de célébrer un amour que Dieu et la société peuvent reconnaître comme légitime, et qui s'accomplit dans le mariage chrétien. Le pèlerinage, mettant à nu les amants et les exposant à l'hostilité du monde, leur permet en définitive de mériter leur bonheur devant Dieu et les hommes. 